\title{
Spontaneous Fracture and Embolization of Port-a-Cath placed Via Subclavian Venous Access: A Case Report
}

\author{
Mikhail CS S Higgins*, Matthew Diamond and Wilson Chavez \\ Boston Medical Center, One Boston Medical Center Place, Boston, MA, 02118, United States \\ *Corresponding author: Mikhail C.S.S. Higgins, Boston Medical Center, One Boston Medical Center Place, Boston, MA, \\ 02118, United States
}

\begin{tabular}{|c|c|}
\hline ARTICLE INFO & ABSTRACT \\
\hline $\begin{array}{l}\text { Received: } \text { April 16, } 2019 \\
\text { Published: } \text { April 26, } 2019\end{array}$ & $\begin{array}{l}\text { The purpose of this article is to present a rare case report of a spontaneous fracture and } \\
\text { embolization of a port-a-cath, known as pinch-off syndrome. This infrequent complication }\end{array}$ \\
\hline $\begin{array}{l}\text { Citation: Mikhail CS S H*, Matthew } \\
\text { D,Wilson C. Mikhail CS S Higgins*, } \\
\text { Matthew Diamond and Wilson } \\
\text { Chavez. Biomed J Sci \& Tech Res } \\
\text { 17(3)-2019. BJSTR. MS.ID.003008. }\end{array}$ & $\begin{array}{l}\text { the primary reasons why surgeons and interventionalists have slowly shifted away from } \\
\text { subclavian venous access to internal jugular venous access when considering both a safe } \\
\text { and reliable access for port placement. Knowledge of this complication is critical to both } \\
\text { operators placing these devices, as well as radiologists reviewing the imaging of such } \\
\text { patients in follow up. }\end{array}$ \\
\hline
\end{tabular}

\section{Introduction}

A port-a-cath, also known as a chemoport, is an Implanted Venous Access Device (ICVAD). Placement of a port-a-cath is performed under maximal sterile barrier precautions in an angiography suite by an interventional radiologist or vascular surgeon or in an operating theater by a general surgeon or surgical subspecialist. Unlike others, these implantable central devices are completely buried underneath the skin with the catheter component extending centrally, ideally terminating in the mid right atrium. They are routinely placed when long term central venous access is required such as for administration of chemotherapy, total parenteral nutrition, blood products or other intravenous medications [1]. The placement of a port is considered low to medium risk with complications occurring early $(<30$ days) and late ( $>30$ days). Pinch-off syndrome, which is a rare and characteristically late occurring complication of port-a-caths, are unique to those placed via a subclavian venous access [2]. The purpose of this article is to describe a case of pinch-off syndrome, namely spontaneous fracture and embolization of a port-a-cath placed via a subclavian venous access.

\section{Case Report}

A 56-year-old man with colon cancer currently undergoing chemotherapy presented to a tertiary medical center with failure

to achieve blood return during attempted access of his port in the chemoinfusion center. The patient had his port placed approximately 11 months prior via the left subclavian vein. It had been used successfully throughout his treatment without any reported prior access challenges. The patient was subsequently referred to interventional radiology for consultation and evaluation of his port. Review of his prior imaging revealed a post placement radiograph as well as a Chest CT obtained 1 month and 5 months post port placement, respectively, with an unremarkable appearing, intact port-a-cath. Review of systems was negative for chest pain, neck pain, shortness of breath, or any other symptoms of note. A focused physical examination revealed no tenderness or erythema regional to the port site. The patient was consented for a port check in the angiography suite. Prior to accessing the port, a routine preassessment scout radiograph was then performed, demonstrating an intact left chest port, but with fractured catheter deep to the left clavicle with an embolized fragment in the right pulmonary arterial vasculature. After discussion of the findings with the referring provider and the patient, informed consent was obtained for explant of the port reservoir and endovascular retrieval of the embolized catheter fragment. The latter was achieved using loop snare technique via common femoral venous access. A new functioning chest port was then placed via internal jugular venous access (Figure $1 \& 2$ ). 


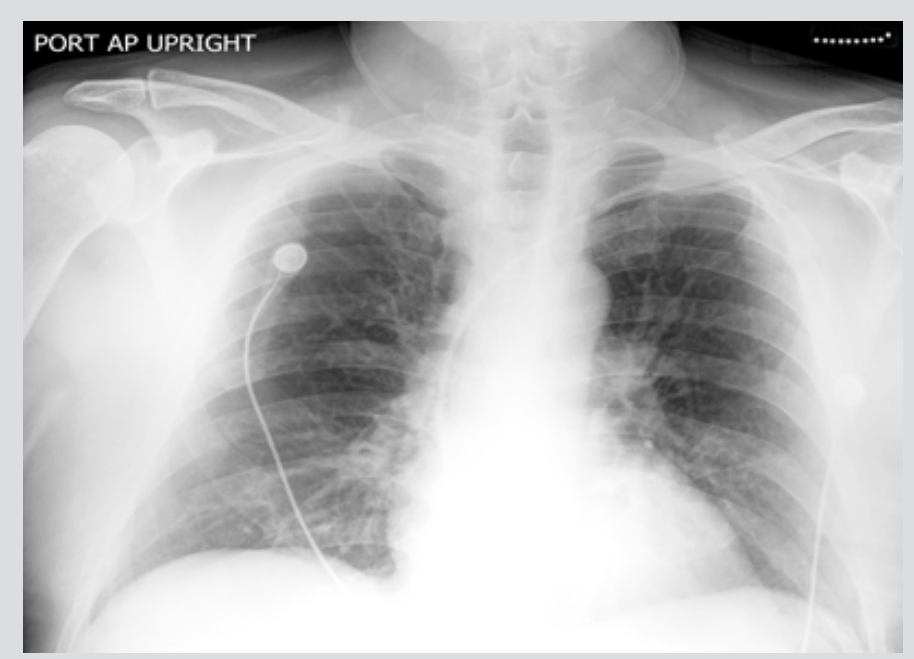

Figure 1: Chest Radiograph performed 1- month after surgical placement of the left chest port via left subclavian venous approach.
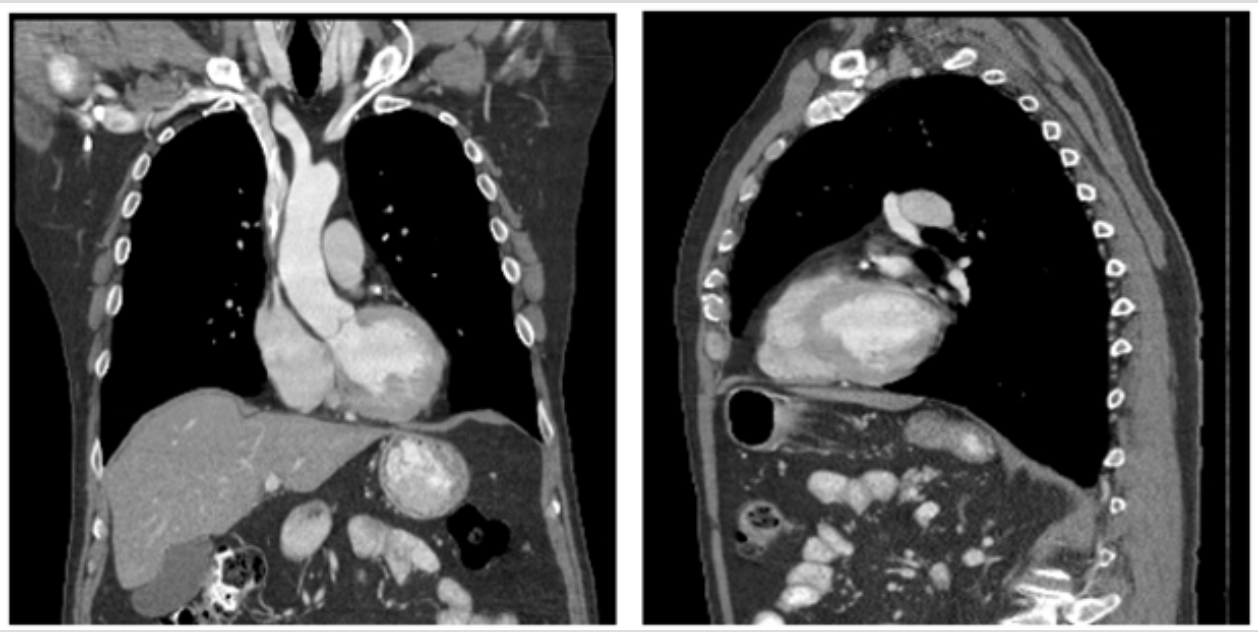

Figure 1: Chest CT performed 5 months post port placement demonstrates intact catheter coursing between the left costoclavicular ligament and subclavius muscle at the level of the first rib and clavicle.

\section{Discussion}

Fragmentation of central venous catheters placed via the subclavian venous route is a rare often late occurring complication $[2,3]$. Historically, the subclavian vein has been the preferred route for placement of central venous catheters such as port-a-caths secondary to use of reliable anatomical landmarks to facilitate swift successful nonequipment dependent venous access [4]. Subclavian venous access is associated with lower probability of catheterassociated infection when compared to both internal jugular and common femoral access [5,6]. However, subclavian venous access is associated with notable complications, including inadvertent arterial access (3.1\%-4.9\%), hematoma formation (1.2-2.1\%), pneumothorax (1.5-3.1\%) and hemothorax (0.4-0.6\%) [5].

Given variations observed in internal jugular venous anatomy, the landmark method has not proved dependable for this site. This has resulted in increased ultrasound-guided internal jugular venous access which has provided a safer vascular access alternative, with higher success, a lower rate of complication, and overall swifter access [4]. When port-a-caths are placed via the subclavian venous route, the catheter component courses between the costoclavicular ligament and subclavius muscle at the level of the first rib and clavicle [4].

The attached catheter is comprised of silicone or polyurethane [7]. These constituent materials are soft and flexible, in order to minimize both its trauma on the endothelium of the superior vena cava or right atrium, as well the catheter's risk for fragmentation. Given repeated cycles of inspiration and expiration, dynamic compressive forces acting on the catheter during its course provokes repetitive stress that causes wear and tear. Over time, fragmentation and tearing of the distressed catheter may result, followed by embolization of the detached fragment. Alternatively, transection of the catheter may also be precipitated by mechanical defects within the catheter, inadvertent damage to the catheter occurring at the point of placement or by needle access, or by an 
additional central venous catheter coursing via a contiguous access [8]. The most common sites for embolization of the distal detached catheter fragment include the pulmonary artery (35\%), right atrium $(27.6 \%)$ and right ventricle $(22 \%)$, the superior vena cava or more peripheral veins such as the thymic vein or hepatic veins (15.4\%) [9].

Associated complications from embolization of catheter fragments include pulmonary thromboembolism, arrhythmias, endocarditis, cardiac perforation and in certain circumstances mechanical outflow obstruction, which may provoke right heart failure [10]. All of these complications may prove fatal [11]. When patients are asymptomatic and the migrated fragment is not recognized, it may become endothelialized, which complicates its removal. Approximately $4.2 \%$ of identified cases which are adherent to the intravascular wall have been reported, prompting surgical consultation [9]. As a result, paying careful attention on routine chest radiographs to the full course of the port-a-cath is recommended to ensure catheter continuity. If an embolized fragment is detected early, prior to its endothelization, it may be retrieved by loop snare technique via percutaneous access by an interventional radiologist, predominantly from a common femoral venous approach [12]. Time to occurrence of pinch-off syndrome may range from months to years. Mirza et al reports an average of 5.3 months and an upper limit of 60 months in a series of 112 reported cases [3].

Mortality from embolization of detached catheter fragments has reportedly ranged between $28 \%$ and $57 \%$ in case reports and series between 1950 and 1980, presumably secondary to distinct constituent catheter components, which have since evolved [8,1315]. Serov et al however, has since reported a mortality of $1.8 \%$ for patients with pinch-off syndrome. There are a few points that have been considered to minimize the risk of pinch off syndrome in central venous catheters placed via the subclavian route. A considerably medial subcutaneous implantation of the port reservoir has been cited as having a high likelihood of increasing the compressive forces exerted by the costoclavicular ligament and the subclavius muscle on the catheter $[1,16,17]$. The lateral insertion technique has also been proposed as a means of preempting catheter-pinch off. However, Kawagishi et al. [18] demonstrates that due to the tightly adherent nature of the subclavius muscle and vein, the lateral insertion technique is unlikely to systematically prevent penetration of the subclavius muscle, which in part contributes to catheter compression and the ensuing pinch-off [18].

Using ultrasound to facilitate real-time guided placement of central venous accesses via the subclavian route has demonstrated a decreased rate of complications relative to the landmark method [15]. While pinch-off is an infrequent complication associated with port-a-caths placed via the subclavian vein, it has not been reported in ports placed via the internal jugular venous access. This is one possible reason why surgeons and interventionalists have slowly shifted away from subclavian venous access to internal jugular venous access when considering both a safe and reliable access for port placement.

\section{References}

1. Funaki B (2002) Central venous access: A primer for the diagnostic radiologist. AJR 179: 309-318.

2. Nostdahl T, Waagsbo NA (2008) Costoclavicular pinching: A complication of long-term central venous catheters. Acta Anaesthesiol Scand 42: 872875 .

3. Mirza B, Vanek VW, Kupensky DT (2004) Pinch-off syndrome: Case report and collective review of the literature. Am Surg 70: 635-644.

4. Jensen MO (2008) Anatomical basis of central venous catheter fracture. Clinical Anatomy 21(2): 106-110.

5. McGee DC, Gould MK (2003) Preventing complications of central venous catheterization. New Engl J Med 348: 1123-1133.

6. Polderman KH, Girbes ARJ (2002) Central venous catheter use. Part 2: Infectious complications. Intensive Care Med 28: 18-28.

7. Rachapalli V, Sharma P, Kumar S, Uthappa MC (2017) Do you know your lines? J Clin Interv Radiol ISVIR 1: 161-167.

8. Morales Victorino N, Damas de los Santos F, Kuri-Ayache M, LopezAguilar C (2015) Pinch-off syndomre. Case report and review of the literature. Gac Med Mex 151: 494-496.

9. Surov A, Wienke A, Carter JM, Stoevesandt D, Behrmann C, et al. (2009) Intravascular embolization of venous catheter -causes, clinical signs, and management: A systematic review. JPEN J Parenter Enteral Nutr 33: 677-685.

10. Unal E, Balci S, Atceken Z, Akpinar E, Ariyurek OM (2017) Nonthrombotic pulmonary artery embolism: imaging findings and review of the literature. AJR 208: 505-516.

11. Bessoud B, de Baere T, Kuoc V, Desruennes E, Cosset MF, et al. (2003) Experience at a single institution with endovascular treatment of mechanical complications caused by implanted central venous access devices in pediatric and adult patients. AJR 180: 527-532.

12. Gablmann A, Kraker S, Gorich J (2001) Percutaneous retrieval of lost or misplaced intravascular objects. AJR 176: 1509-1513.

13. Busch JD, Vens M, Herrmann J, Adam G, Ittrich H (2017) Material failure of silicone catheter lines: a retrospective review of partial and complete ruptures in 553 patients. AJR 208: 464-469.

14. Marcy PY, Lacout A, ElHajjam M (2017) Failure of silicone catheter lines: Where, when and why. AJR 209: W49-W51.

15. Wildgruber M (2017) Catheter rupture is due to material fatigue of the implanted silicone catheters. AJR 209: W198.

16. Krutchen AE, Bjarnason H, Stackhouse DJ, Nazarian GK, Magney JE, et al. (1996) The mechanisms of positional dysfunction of subclavian venous catheters. Radiology 200: 159-163.

17. Hinke DH, Zandt-Stastny DA, Goodman LR, Quebbeman EJ, Krzywda EA, et al. (1990) Pinch-off syndrome: A complication of implantable subclavian venous access devices. Radiology 177: 353-356.

18. Kawagishi K, Tokumin J, and Lefor AK (2016) The relationship of the subclavius muscle with relevance to venous cannulation below the clavicle. Anesthesiol Res Pract 2016.

19. Fragou M, Gravvanis A, Dimitriou V, Papalois A, Kouraklis G, et al. (2011) Real-time ultrasound-guided subclavian vein cannulation versus the landmark method in critical care patients: a prospective randomised study. Crit Care Med 39: 1607-1612. 


\section{ISSN: 2574-1241}

DOI: 10.26717/BJSTR.2019.17.003008

Mikhail C.S.S. Higgins. Biomed J Sci \& Tech Res

(c) 9 This work is licensed under Creative

Submission Link: https://biomedres.us/submit-manuscript.php

$\begin{array}{ll}\text { BIOMEDICAL } & \text { Assets of Publishing with us } \\ \text { RESEARCHES } & \text { - Global archiving of articles } \\ \text { - Immediate, unrestricted online access }\end{array}$

